\title{
Identificación de la Respuesta de Estructuras con Aislamiento de Base durante Terremotos de Gran Magnitud
}

\author{
O. A. Z. Sotomayor ${ }^{a}$, C. A. V. Cardoso ${ }^{b}$ y J. F. Tisza C ${ }^{c}$
}

\section{RESUMEN}

El presente trabajo muestra los resultados iniciales de la aplicación de técnicas de identificación continua en el análisis estructural de edificaciones sometidas a acciones sísmicas de gran intensidad. Resultados de simulaciones numéricas de una estructura real con tres grados de libertad y aislamiento de base, perturbada con datos reales del Terremoto de Pisco, muestran que el modelo identificado en el primer modo por encima de la base captura las principales características dinámicas del sistema, indicando que la respuesta estructural es dominada por la formal modal de la base.

Palabras clave: Identificación de sistemas, Respuesta sísmica, Análisis estructural, Sistemas vibracionales.

\begin{abstract}
The present work, show the initial results of the application from techniques for continuous identification in the structural analysis on an construction submitted to seismic actions of great intensity. The results from applied numerical simulations to real structure with three degrees of freedom isolated in basement, it is submitted to disturbances with real data of the Earthquake of Pisco, showing that the model identified in the first floor over the base captures the main dynamic characteristics of the system, indicating that structural answer is dominated by the modal form of the base. Key Words: Identification of systems, seismic response, structural analysis, vibration systems
\end{abstract}

\section{INTRODUCCIÓN}

Una de las más excitantes áreas de aplicación de la identificación de sistemas es con referente a la obtención de modelos matemáticos de estructuras a partir de sus respuestas a terremotos de gran magnitud. La análisis de estos modelos y mediciones periódicas permiten la detección de daños en la estructura y la evaluación del nivel de protección y seguridad, incluyendo su contenido material y ocupantes humanos, lo cual es sin lugar a dudas una prioridad mundial [1], llevando en cuenta terremotos recientes ocurridos en Indonesia (2006), Perú (2007) y China (2008).

Innúmeros trabajos de identificación de sistemas estructurales han aparecido en la literatura, abarcando diversas técnicas de identificación y diversos tipos de modelos; ver por ejemplo [2],[3],[4],[5],[6],[7],[8]. Un extenso review sobre técnicas de identificación no lineal en estos sistemas estructurales es dado por [9]. La mayoria de estos trabajos tienen como finalidad obtener un modelo modal o un modelo físico de la estructura. El modelo modal define a respuesta dinámica de estructuras en función de frecuencias de resonancia (frecuencia natural no amortiguada), relaciones de amortiguamiento y formas modales. El modelo físico o espacial, es caracterizado por las matrices de masa, amortiguamiento y rigidez del sistema estructural.
El objetivo del presente trabajo es identificar un modelo continuo que represente la respuesta dinámica de una estructura de 3 pisos con aislamiento de base. Sistemas de aislamiento de base han recibido considerable atención para protección antisísmica de estructuras, debido a su sencillez, bajo costo, eficacia y facilidad de reparación en caso de daño.

En el presente caso, la estructura seleccionada es sometida a perturbaciones correspondientes a los registros del Terremoto de Pisco (2007), que con una magnitud de 7.9 Mw, fue uno de los mas grandes terremotos ocurridos en el Perú en los últimos 100 años. El modelo obtenido es usado para estimar los parámetros modales dominantes del sistema.

\section{ANALISIS Y SIMULACION DEL SISTEMA}

\section{Sistema Estructural}

Se considera una estructura de edificación de tres pisos con aislamiento de base, como mostrado en la Fig. 1. El aislamiento de base actúa como un sistema de control pasivo, el cual desacopla parcialmente la estructura del movimiento del terreno mediante mecanismos que
(a)Dr. O. A. Z. Sotomayor trabaja en el Núcleo de Ingeniería Eléctrica (NEL), Centro de Ciencias Exactas y Tecnología, Universidad Federal de Sergipe (UFS), São Cristóvão-SE, Brasil (e-mail: oscar@pqi.ep.usp.br ). (b) Dr. C. A. V. Cardoso trabaja en el NEL de la UFS, São Cristóvão-SE, Brasil (e-mail: cvcardoso@ufs.br ).
(c)Ing. J. F. Tisza C. trabaja en la Facultad de Ingeniería Electrónica y Mecatrónica (FIEM), Universidad Tecnológica del Perú (UTP) y Facultad de Ingeniería Eléctrica Electrónica(FIEE), Universidad Nacional de Ingeniería (UNI),Lima, Perú (e-mail: jitisza@gmail.com ). 
incrementan la flexibilidad del sistema estructura-base y, al mismo tiempo, producen un amortiguamiento apropiado [10]. En caso de terremotos, este sistema absorbe parte de la energía inducida por el terreno, reduciendo el desplazamiento relativo entre elementos estructurales.

\section{Modelo Dinámico}

La estructura tiene cuatro grados de libertad en la dirección horizontal. Dado que el efecto destructivo de los terremotos es producto de la vibración horizontal, en este estudio los grados de libertad son asumidos para ser solamente en esta dirección. El movimiento de la estructura se describe mediante un vector $x(t)$ que representa el desplazamiento horizontal -según sus grados de libertad respecto a un sistema de referencia inercial-, debido a una fuerza de excitación $F_{d}(t)$ producida por un movimiento sísmico horizontal. Así, la ecuación de movimiento del sistema es dado por:

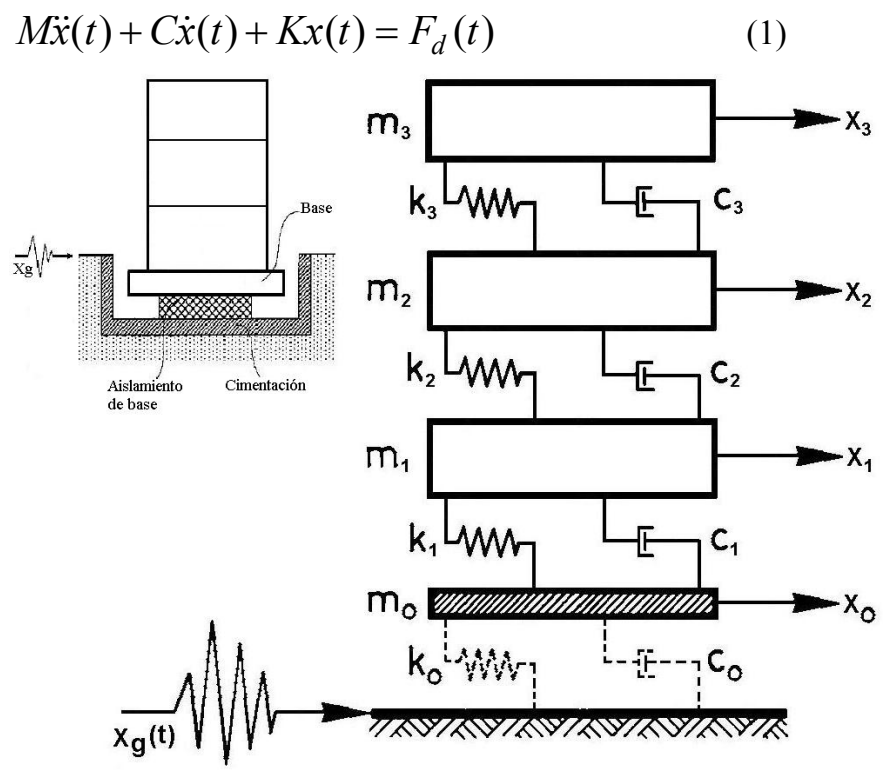

Fig. 1. Modelo dinámico de una estructura de tres pisos con aislamiento de base.

con:

$x(t)=\left[\begin{array}{llll}x_{0}(t) & x_{1}(t) & x_{2}(t) & x_{3}(t)\end{array}\right]^{T}$

$F_{d}(t)=\left[\begin{array}{llll}-\left(c_{0} \dot{x}_{g}(t)+k_{0} x_{g}(t)\right) & 0 & 0 & 0\end{array}\right]^{T}$

$M=\left[\begin{array}{cccc}m_{0} & 0 & 0 & 0 \\ 0 & m_{1} & 0 & 0 \\ 0 & 0 & m_{2} & 0 \\ 0 & 0 & 0 & m_{3}\end{array}\right]$

$C=\left[\begin{array}{cccc}c_{0}+c_{1} & -c_{1} & 0 & 0 \\ -c_{1} & c_{1}+c_{2} & -c_{2} & 0 \\ 0 & -c_{2} & c_{2}+c_{3} & -c_{3} \\ 0 & 0 & -c_{3} & c_{3}\end{array}\right]$
$K=\left[\begin{array}{cccc}k_{0}+k_{1} & -k_{1} & 0 & 0 \\ -k_{1} & k_{1}+k_{2} & -k_{2} & 0 \\ 0 & -k_{2} & k_{2}+k_{3} & -k_{3} \\ 0 & 0 & -k_{3} & k_{3}\end{array}\right]$

donde $x_{i}, m_{i}, c_{i}$ y $k_{i}$ son el desplazamiento, la masa, el amortiguamiento y la rigidez en cada piso $i \quad(=1,2,3)$, respectivamente, $x_{0}$ y $m_{0}$ son el desplazamiento y la masa de la base, $c_{0}$ y $k_{0}$ son el amortiguamiento y la rigidez de los aisladores y $x_{g}$ es el desplazamiento del terreno inducido por el terremoto. Los parámetros del sistema pertenecen a un edificio real y son listados en la Tabla I, los cuales son tomados de [11].

\section{Parámetros Modales}

El modelo descrito por (1) puede ser transformado en la siguiente ecuación de movimiento en espacio de estado:

$$
\dot{z}(t)=A z(t)+B(t)
$$

TABLA I

PARÁMETROS DEL SISTEMA ESTRUCTURAL

\begin{tabular}{lrlr}
\hline \hline \multicolumn{1}{c}{ Símbolo } & \multicolumn{1}{c}{ Valor } & Símbolo & \multicolumn{1}{c}{ Valor } \\
\hline$m_{0}$ & $450000 \mathrm{~kg}$ & $k_{3}$ & 250000000 \\
$m_{1}=m_{2}=m_{3}$ & $345000 \mathrm{~kg}$ & $c_{0}$ & $26170 \mathrm{~N} \mathrm{~s} / \mathrm{m}$ \\
$k_{0}$ & 18050000 & $c_{1}$ & $467000 \mathrm{~N} \mathrm{~s} / \mathrm{m}$ \\
$k_{1}$ & $\mathrm{~N} / \mathrm{m}$ & & \\
$k_{2}$ & 326000000 & $c_{2}$ & $410000 \mathrm{~N} \mathrm{~s} / \mathrm{m}$ \\
\hline
\end{tabular}

donde:

$$
\begin{aligned}
& z(t)=\left[\begin{array}{ll}
x(t) & \dot{x}(t)
\end{array}\right]^{T} \\
& A=\left[\begin{array}{cc}
0 & I \\
-M^{-1} K & -M^{-1} C
\end{array}\right], B(t)=\left[\begin{array}{c}
0 \\
M^{-1} F_{d}(t)
\end{array}\right]
\end{aligned}
$$

Las frecuencias de resonancia $\omega_{n}$ y las relaciones de amortiguamiento $\xi$ son obtenidos a partir de un eigenanálisis de la matriz $A$ en (2). Considerando que los autovalores de la matriz $A$ tienen la forma $s_{i}=\alpha_{i}+j \beta_{i}$ $(i=1,2, \cdots, r ; r=\operatorname{rank}(A)), \quad$ las frecuencia de resonancia y las relaciones de amortiguamiento modales son [4]:

$$
\begin{aligned}
& \omega_{n, i}=\sqrt{\alpha_{i}+\beta_{i}} / 2 \pi \quad(\mathrm{Hz}) \\
& \xi_{i}=-\alpha_{i} / \omega_{i} \quad(\%)
\end{aligned}
$$

Usando (3a) y (3b) y los datos de la Tabela I, las frecuencias de resonancia del sistema son $0.54,3.37,6.27$ y $8.42 \mathrm{~Hz}$ y las relaciones de amortiguamiento son 0.016 , 
$0.095,0.175$ y $0.238 \%$, para la base y los pisos 1,2 y 3 , respectivamente.

\section{Respuesta de la Estructura al Terremoto de Pisco}

\section{Terremoto de Pisco}

"El Cinturón de Fuego del Pacífico" concentra la mayor zona de convergencia y subducción de placas tectónicas de la Tierra, además de una actividad volcánica constante, a las que se le puede asociar el $90 \%$ de la sismicidad total del planeta. Ubicado dentro de este Cinturón, el Perú se encuentra afectado por la subducción de la placa de Nazca (que se desplaza hacia el Este) bajo la sudamericana (que se desplaza hacia el Oeste), con una velocidad relativa de 7-8 cm/año [12]. Este proceso es responsable de la ocurrencia de los sismos que con diversas magnitudes se producen frente a la línea de la costa y a profundidades menores a 60 $\mathrm{km}$.

El 15 de Agosto de 2007, a las 18h 40m 58 (hora local), la zona sur de la región central del Perú, fue afectado por un fuerte sismo que alcanzo una magnitud de 7.0 ML (escala de Richter) y $7.9 \mathrm{Mw}$ (escala magnitud de momento), con epicentro en $13.49^{\circ}$ latitud Sur y $76.85^{\circ}$ longitud Oeste, es decir a $60 \mathrm{~km}$ al Oeste de la ciudad de Pisco, razón por la cual fue denominado "Terremoto de Pisco" (ver mapa en la Fig. 2).

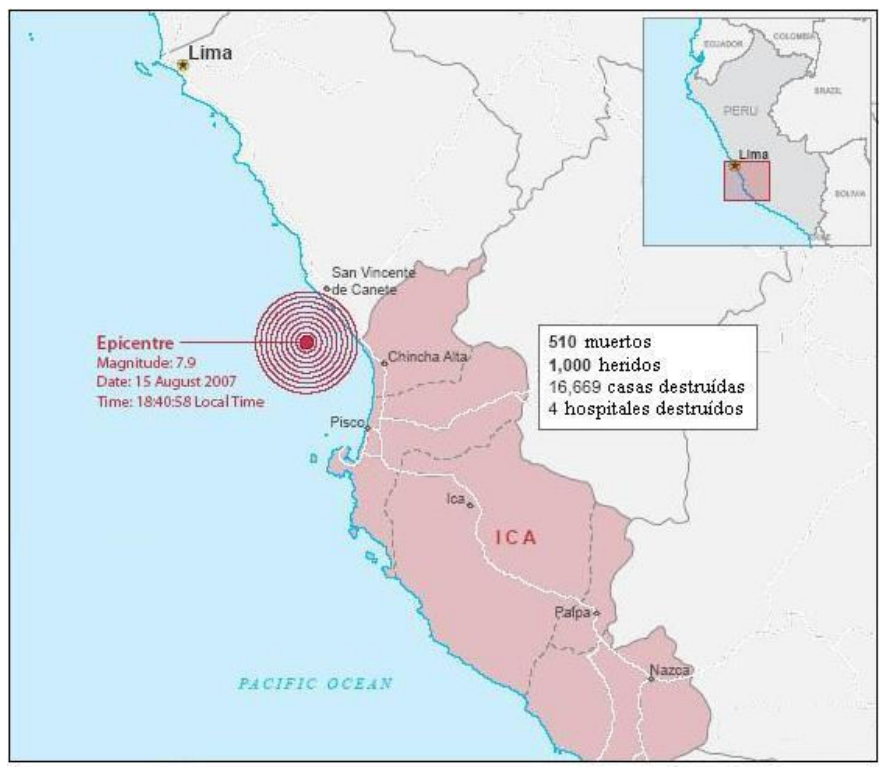

Fig. 2. Localización del Terremoto de Pisco.

El Terremoto de Pisco produjo daños importantes en un gran número de viviendas de la ciudad de Pisco (aproximadamente el $80 \%$ ) y menos en localidades aledañas, donde se evaluaron intensidades de VI-VII MM (escala de Mercalli modificada). Este terremoto se constituye como uno de los mas grandes ocurridos en esta región en los últimos 100 años y se caracterizo por presentar gran duración (aproximadamente 210 segundos) y la ocurrencia de dos frentes de onda (o frentes de ruptura) [13].

La Fig. 3 presenta el registro de la aceleración del suelo correspondiente a la componente transversal (EO) para el Terremoto de Pisco, obtenido por el acelerógrafo de la estación UNSLG (ICA-2, $138 \mathrm{~km}$ de distancia epicentro-estación en dirección SE y $409 \mathrm{msnm}$ ) del CISMID (Centro Peruano-Japonés de Investigaciones Sísmicas y Mitigación de Desastres), con un tiempo de muestreo de $T_{s}=0.01 \mathrm{~s}$. En el registro de aceleración se destaca la presencia de las dos frentes de onda que llegan con una diferencia de tiempo de 65 segundos aproximadamente. El primero identificado como R1 presenta una aceleración máxima de $272.2 \mathrm{~cm} / \mathrm{s}^{2}$ y el segundo, R2 de $175.08 \mathrm{~cm} / \mathrm{s}^{2}$. La Fig. 3 también muestra los registros de velocidad y desplazamiento del suelo obtenidos por integración numérica por el método de Simpson a partir de los datos de la aceleración.

\section{Simulación de la Respuesta de la Estructura}

Los registros del Terremoto de Pisco (componente EO) son usados como entadas de excitación a la estructura. En la práctica, no todos los grados de libertad son monitoreados para identificación experimental de la respuesta dinámica de una estructura. Sin embargo, en el presente caso, se considera que cada modo de la estructura está equipado con un aceleró-grafo, los cuales son usados como sensores. La Fig. 4 presenta los desplazamientos absolutos y relativos de la estructura.
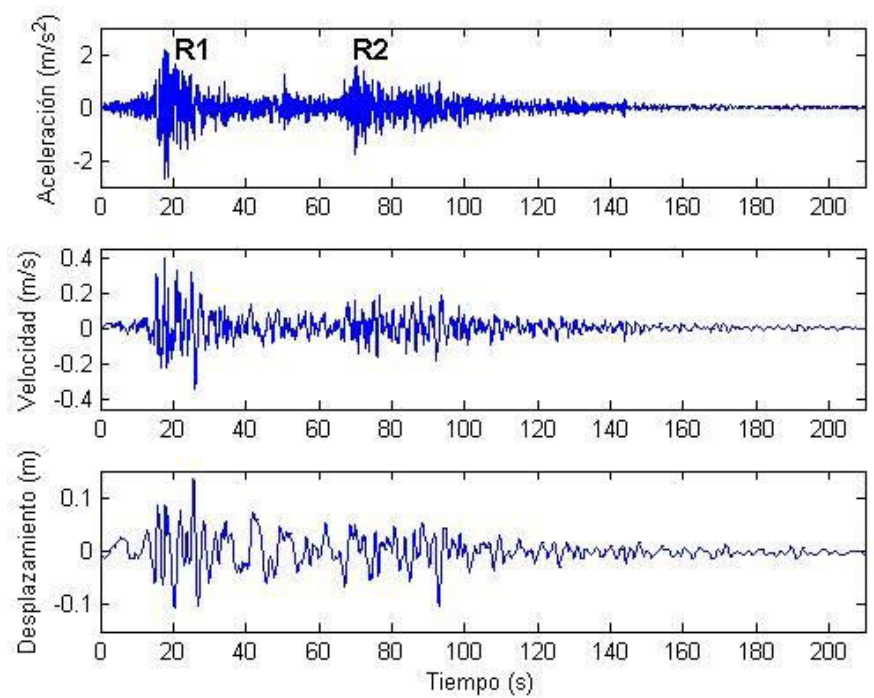

Fig. 3. Registros del terremoto de Pisco (componente EO).

En la Fig. 4, los desplazamientos absolutos fueron inferidos por integración on-line de los datos proporcionados por los acelerógrafos. Los desplazamientos relativos de los pisos son en relación al desplazamiento de la base. Estos desplazamientos dan una visión de los esfuerzos y tensiones que se producen en cada piso de la estructura, por lo que proporciona una medida del daño sufrido por dicha estructura. Como puede ser observado, los desplazamientos relativos de os pisos superiores respecto a la base son bien reducidos.

La Fig. 4 también muestra que la forma de operación estructural es dominada por un modo particular en una determinada frecuencia de resonancia. 

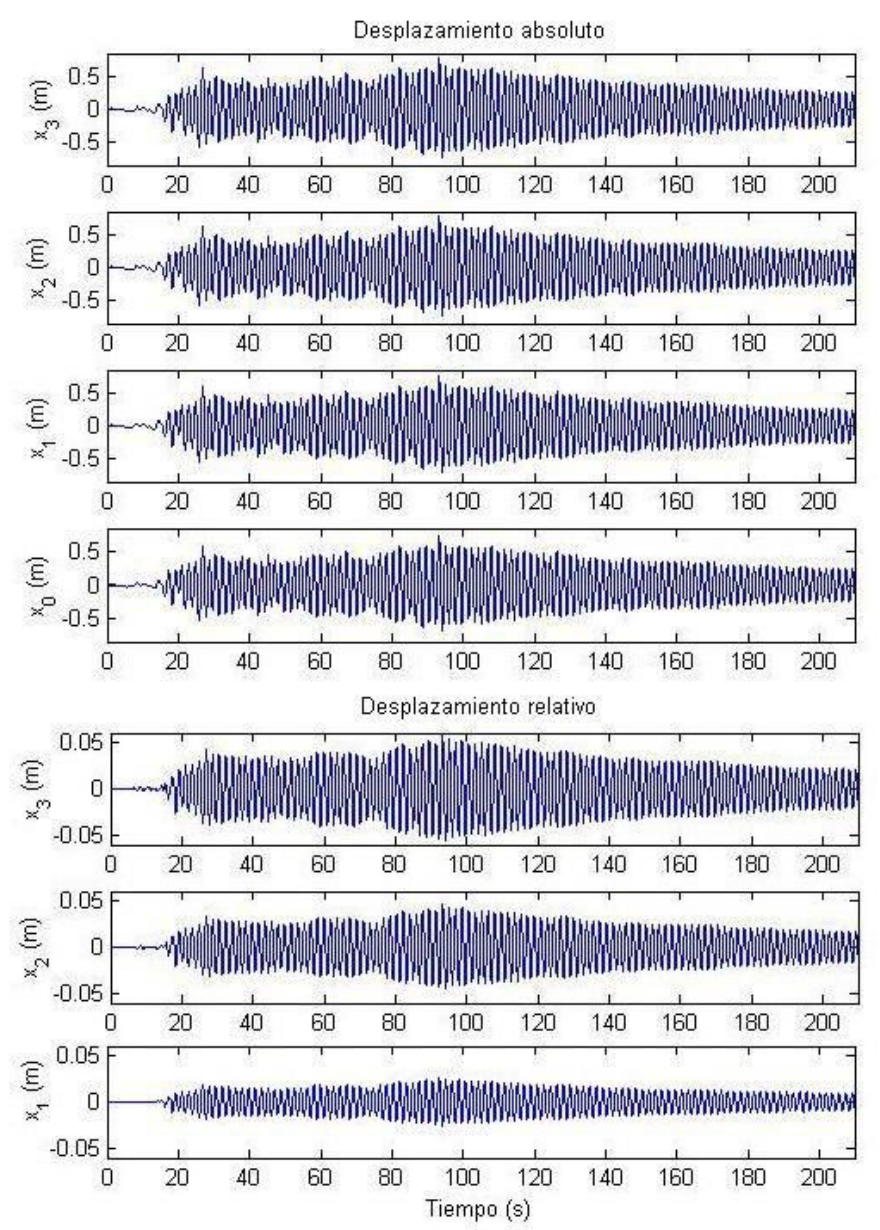

Fig. 4. Desplazamientos absolutos y relativos de la estructura.

\section{Procedimiento de Identificación}

Como caso de aplicación del presente estudio, la identificación será realizada en el primer modo por encima de la base. La selección fue hecha tomando en cuenta que durante un terremoto la máxima conjunción de fuerzas inter-pisos sucede en el primer piso, donde es esperado el mayor efecto destructivo del fenómeno.

Un modelo adecuado para describir la salida, desplazamiento en el piso 1 , es una ecuación de segunda orden de la forma:

$$
x_{1}(s)=\underbrace{\left(\frac{b_{0}}{s^{2}+a_{1} s+a_{2}}\right)}_{G_{1}(s)} x_{g}(s)
$$

Por tanto, el problema de identificación se reduce a obtener la función de transferencia $G_{1}(s)=B(s) / F(s)$ usando datos de los desplazamientos de entrada $x_{g}$ y salida $x_{1}$, del sistema.

Como es usual en todo procedimiento de identificación, los datos son pre-tratados antes de ser usados, i.e. remoción de su valor medio, eliminación de tendencias lineales y filtrado. Una parte de los datos correspondiente a la frente de onda R1 (primeras 6500 muestras) es usado para identificación y otra parte de datos correspondiente a la frente de onda R2 (siguientes 6500 muestras) es usado para validación cruzada del modelo. La identificación es realizada usando el algoritmo de identificación SRIVC del CONTSID toolbox [14], que presenta nuevos métodos de identificación continua a partir de datos muestreados. La rutina de identificación es escrita de la siguiente manera:

$\mathrm{z}=$ iddata $\left(x_{1}, x_{g}, T_{s}\right)$;

$\mathrm{G}=\operatorname{srivc}\left(z,\left[\begin{array}{ll}1 & 2\end{array}\right]\right)$;

lo que genera los siguientes resultados:

CT IDPOLY model: $\mathrm{G}(\mathrm{s})=[\mathrm{B}(\mathrm{s}) / \mathrm{F}(\mathrm{s})]$

$\mathrm{B}(\mathrm{s})=-11.11$

$\mathrm{F}(\mathrm{s})=s^{2}+0.01736( \pm 2.087 \mathrm{e}-5) s+11.63( \pm 3.691 \mathrm{e}-5)$

\section{Estimated using SRIVC}

Loss function 5.08272e-6 and FPE 5.08742e-6

Los valores entre paréntesis son las desviaciones estándar de los asociados parámetros identificados. La frecuencia de resonancia y la relación de amortiguamiento de este modelo son $0.5427 \mathrm{~Hz}$ y 0.0156 $\%$, respectivamente, lo cual significa que la forma de operación estructural se aproxima de la forma modal de la base.
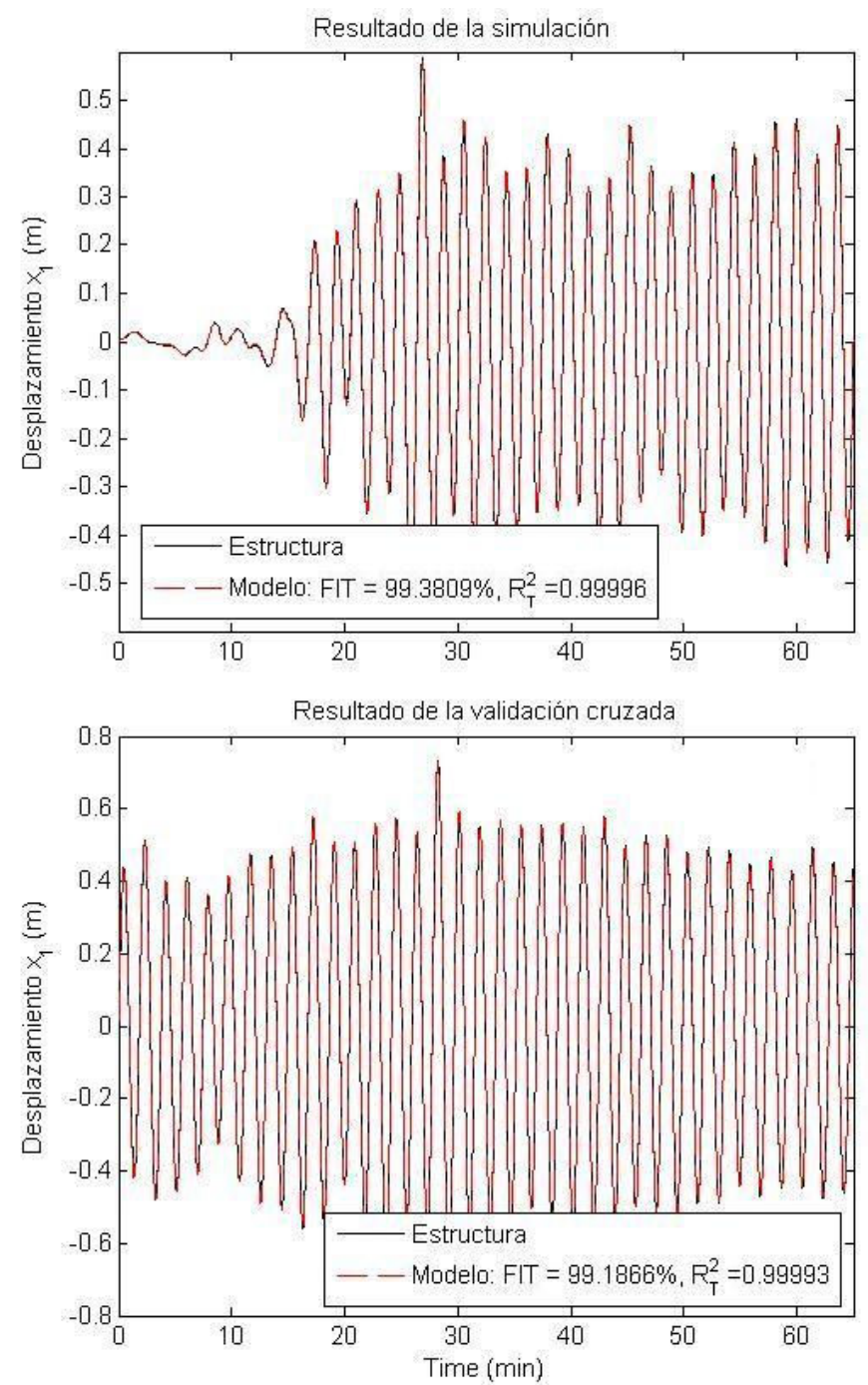

Fig. 5. Resultados de la simulación e validación cruzada del modelo 


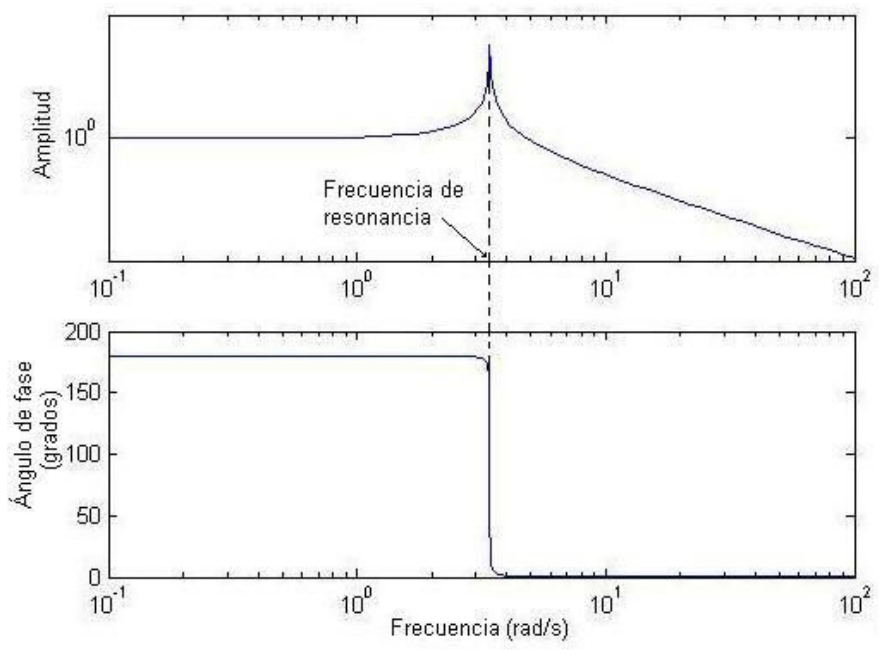

Fig. 6. Respuesta en frecuencia del modelo identificado.

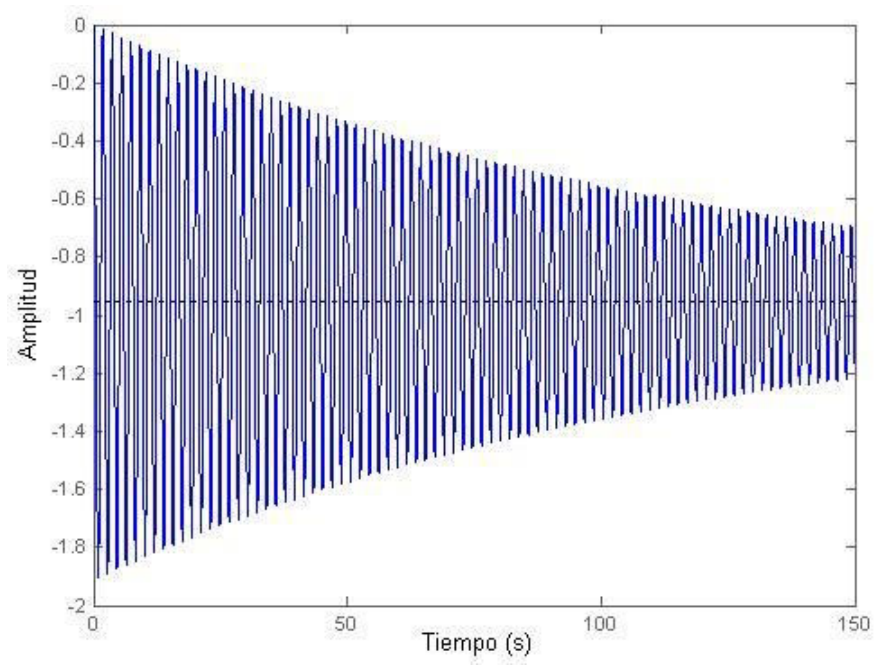

Fig. 7. Respuesta al escalón unitario del modelo identificado.

La Fig. 5 muestra los resultados de la simulación (identificación) y validación cruzada del modelo. Una rápida y simple inspección visual de estos gráficos e informaciones de los índices de desempeño (coeficiente de ajuste FIT e coeficiente de determinación $R_{T}^{2}$ ) indican que el modelo identificado reproduce muy bien las dinámicas dominantes de la estructura. La Fig. 6 muestra la respuesta en frecuencia usando el diagrama de Bode y la Fig. 7 la respuesta al escalón unitario del modelo identificado, los cuales claramente indican el comportamiento sub-amortiguado del sistema.

\section{CONCLUSIONES}

Este trabajo propone identificar la respuesta de una estructura de 3 pisos con aislamiento de base durante una excitación sísmica de grande magnitud. La aplicación de los datos de registro del Terremoto de Pisco, muestra que el control pasivo por aislamiento de base es efectivo para reducir los desplazamientos relativos de los pisos respecto a la base. Sin embargo, su capacidad para reducir la respuesta estructural a un sismo de gran magnitud es limitada, haciendo que la operación estructural sea dominada por un modo particular en una determinada frecuencia de resonancia.

El procedimiento de identificación es realizado asumiendo un modelo continuo de segunda orden de la respuesta de la estructura en el primer piso y usando el toolbox de identificación continua CONTSID. Los resultados obtenidos indican que el modelo identificado representa muy bien las características dinámicas del sistema cuya respuesta estructural es dominada por la forma modal de la base.

Posteriores trabajos serán dirigidos a la consolidación de la metodología y factibilidad de aplicación en estructuras reales, identificación de estructuras con mayor grados de libertad y al uso de los resultados en el proyecto de sistemas de control activo de estructuras. 


\section{REFERENCIAS BIBLIOGRÁFICAS}

[1] B.F. Spencer y M.K. Sain, "Controlling buildings: A new frontier in feedback", IEEE Control Systems Magazine, vol. 17, n. 6, pp. 19-35, 1993.

[2] H. Lus, R. Betti y R.W. Longman, "Identification of linear structural systems using earthquake-induced vibration data", Earthquake Engineering and Structural Dynamics, vol. 28, n. 11, pp. 1449-1467, 1999.

[3] W.J. Ko y C.F. Hung, "Extraction of structural systems matrices from an identified state-space system using the combined measurements of DVA", Journal of Sound and Vibration, vol. 249, n. 5, pp. 995-970, 2002.

[4] C.-H., Hung, W.-J. Ko y Y.-T. Peng, "Identification of modal parameters from measured input and output data using a vector backward auto-regressive with exogeneous model", Journal of Sound and Vibration, vol. 276, n. 3-5, pp. 995970, 2002.

[5] T. Furukawa, M. Ito, K. Izawa y M.N. Noori, "System identification of base-isolated building using seismic response data”, Journal of Engineering Mechanics, vol. 131, n.3, pp. 268-275, 2005.

[6] P.R.G. Kurka y H.N. Cambraia,"Application of multivariable input-output subspace identification technique in structural analysis", Journal of Sound and Vibration, vol. 312, n. 3, pp. 461-475, 2008.

[7] K. Beyen, "Structural identification for post-earthquake safety analysis of the Fatih mosque after the 17 August 1999 Kocaeli earthquake", Engineering Structures, to be published.

[8] D.M. Siringoringo y Y. Fujino, "System identification of suspension bridge from ambient vibration response", Engineering Structures, to be published.

[9] G. Kerschen, K. Worden, A.F. Vakakis y J.-C Golinval, "Past, present and future of nonlinear system identification in structural dynamics", Mechanical Systems and Signal Processing, vol. 20, n.3, pp. 505-592, 2006.

[10] N.M. Amaya, "Sistemas de control pasivos y activos de aislamiento de base para edificios sometidos a acciones sísmicas", Revista Ingeniería \& Desarrollo, Universidad del Norte, Colombia, n.14, pp. 60-92, 2003.

[11]R. Guclu, "Sliding mode and PID control of a structural system against earthquake", Mathematical and Computer Modelling, vol. 44, n. 1-2, pp. 210-217, 2006.

[12]E. Norabuena, T. Dixon, S. Stein y C. Harrison, "Decelerating Nazca-South America and Nazca-Pacific plate motions", Geophysical Research Letters, vol. 26, n.22, pp. 3405-3408, 1999.

[13] H. Tavera, I. Bernal y H. Salas, El sismo de Pisco del 15 de agosto, 2007 (Mw=7.9 Mw)", Instituto Geofísico del Peru, Lima, 2007.

[14]H. Garnier, M. Gilson, T. Bastogne y M. Mensler, "The CONTSID toolbox: A software support for continuous-time data-based modeling", in Identification of continuous-time models from sampled data, Garnier, H. y L. Wang, Eds., Berlin: Springer-Verlag, 2008.

E-mail: jtisza@gmail.com 